OPEN ACCESS

Edited by:

Heike Folsch,

Northwestern University,

United States

Reviewed by:

James Alan Marrs,

Indiana University, United States

Elaine L. Bearer,

University of New Mexico Health

Sciences Center, United States

*Correspondence:

Francesca Finetti

finetti4@unisi.it

Cosima T. Baldari

cosima.baldari@unisi.it

Specialty section:

This article was submitted to

Membrane Traffic,

a section of the journal

Frontiers in Cell and Developmental

Biology

Received: 07 August 2019 Accepted: 06 November 2019

Published: 19 November 2019

Citation:

Finetti F, Capitani N and

Baldari CT (2019) Emerging Roles of the Intraflagellar Transport System in the Orchestration of Cellular

Degradation Pathways.

Front. Cell Dev. Biol. 7:292.

doi: 10.3389/fcell.2019.00292

\section{Emerging Roles of the Intraflagellar Transport System in the Orchestration of Cellular Degradation Pathways}

\author{
Francesca Finetti*, Nagaja Capitani and Cosima T. Baldari*
}

Department of Life Sciences, University of Siena, Siena, Italy

Ciliated cells exploit a specific transport system, the intraflagellar transport (IFT) system, to ensure the traffic of molecules from the cell body to the cilium. However, it is now clear that IFT activity is not restricted to cilia-related functions. This is strikingly exemplified by the observation that IFT proteins play important roles in cells lacking a primary cilium, such as lymphocytes. Indeed, in T cells the IFT system regulates the polarized transport of endosome-associated $T$ cell antigen receptors and signaling mediators during assembly of the immune synapse, a specialized interface that forms on encounter with a cognate antigen presenting cell and on which $T$ cell activation and effector function crucially depend. Cellular degradation pathways have recently emerged as new extraciliary functions of the IFT system. IFT proteins have been demonstrated to regulate autophagy in ciliated cells through their ability to recruit the autophagy machinery to the base of the cilium. We have now implicated the IFT component IFT20 in another central degradation process that also controls the latest steps in autophagy, namely lysosome function, by regulating the cation-independent mannose-6-phosphate receptor (Cl-MPR)-dependent lysosomal targeting of acid hydrolases. This involves the ability of IFT20 to act as an adaptor coupling the CI-MPR to dynein for retrograde transport to the trans-Golgi network. In this short review we will discuss the emerging roles of IFT proteins in cellular degradation pathways.

Keywords: intraflagellar transport, T cell, degradation pathways, autophagy, lysosome

\section{INTRODUCTION}

Primary cilium assembly and function crucially relies on the intraflagellar transport (IFT) system. This multimolecular machinery, first described by Rosenbaum's lab in the 1990s (Kozminski et al., 1995), allows for the crossing of proteins destined for the cilium through the physical barrier set by the transition zone at the base of the cilium and for their transport along the ciliary axoneme (Pedersen and Rosenbaum, 2008; Gonçalves and Pelletier, 2017). IFT depends on a wide array of IFT proteins that are organized into two complexes: the anterograde IFT-B complex, which interacts with kinesin to regulate the transport of molecules from the base to the tip of the cilium, and the retrograde IFT-A complex, which is required for the retrograde transport to the basal body through the interaction with dynein (Taschner and Lorentzen, 2016; Prevo et al., 2017). 
Both cytosolic and membrane-associated proteins are delivered to the cilium by the IFT system, which interfaces with a complex network of vesicular trafficking regulators (Pedersen et al., 2016).

Interestingly, evidence generated over the last 10 years indicates that the activity of the IFT system is not restricted to the primary cilium but contributes to key extraciliary functions, including cell cycle progression (Robert et al., 2007), mitotic spindle orientation (Jonassen et al., 2008; Delaval et al., 2011; Borovina and Ciruna, 2013), cleavage furrow formation (Qin et al., 2007; Wood et al., 2012; Taulet et al., 2017), microtubule and actin cytoskeleton dynamics (Bizet et al., 2015; Wang et al., 2016; Nishita et al., 2017), intracellular vesicular trafficking (Hsiao et al., 2012), and autophagy (Pampliega et al., 2013). The reader is referred to the thought-provoking review by Hua and Ferland (2018) for details regarding extraciliary roles for ciliary proteins. Consistent with these extraciliary functions, imaging studies have provided evidence that IFT proteins are localized not only at the base of the cilium and along the ciliary axoneme, but also in the cell cytoplasm. This is exemplified by IFT20, a large pool of which is associated with the Golgi complex and post-Golgi endosomal compartments (Follit et al., 2006, 2008; Finetti et al., 2009), and several other IFT proteins that have been detected in association with cytoplasmic vesicles in photoreceptor cells and in the postsynaptic terminals of non-ciliated secondary neurons (Sedmak and Wolfrum, 2010). Whether the extraciliary functions of the IFT system are carried out by IFT complexes with the same composition as those implicated in ciliogenesis remains to date a major open question.

The association of IFT proteins with endosomal compartments bears witness to their implication in intracellular vesicular trafficking, a role which had been initially proposed by Jékely and Arendt (2006) based on structural similarities with components of membrane coats revealed by bioinformatic analyses (Satir et al., 2008). We provided the first evidence of a cilium-independent, vesicular trafficking-related function of the IFT system in T lymphocytes which, similar to other hematopoietic cells, do not have a primary cilium (Finetti et al., 2009). We showed that IFT20 acts in concert with other IFT proteins to promote the assembly of the $\mathrm{T}$ cell immune synapse (Finetti et al., 2009), a specialized signaling platform that forms when a $\mathrm{T}$ cell encounters an antigen presenting cell that displays a specific peptide ligand bound to $\mathrm{MHC}$ and on which $\mathrm{T}$ cell activation crucially depends (Dustin and Choudhuri, 2016). This function involves the ability of IFT20 to interface with trafficking regulators and molecular motors to allow for the polarized delivery to the synaptic membrane of $\mathrm{T}$ cell antigen receptors and membrane-bound signaling mediators associated with recycling endosomes (Finetti et al., 2014; Onnis et al., 2016; Vivar et al., 2016; Figure 1B). We refer the reader to a recent review summarizing our current understanding of how $\mathrm{T}$ cells exploit IFT and other ciliary proteins for immune synapse formation (Cassioli and Baldari, 2019). Here, we will describe the emerging roles of the IFT system in intracellular degradation pathways, with a focus on recent findings by our lab that identify a new, trafficking-related function of IFT20 in the biogenesis of lysosomes.

\section{THE IFT SYSTEM IN CELLULAR DEGRADATION PATHWAYS}

Protein degradation through the ubiquitin-proteasome system (UPS) is essential for the assembly and resorption of the primary cilium (Kasahara et al., 2014). Among the targets of the UPS are centrosome-associated proteins, such as CSPP1, CP110, and CEP97, that negatively regulate ciliogenesis (Spektor et al., 2007; Tuz et al., 2014; Boukhalfa et al., 2019). Additionally, ubiquitin and ubiquitin ligases accumulate in flagella during resorption in the model organism Chlamydomonas reinhardtii, particularly in IFT mutants, indicating the UPS is active during cilia disassembly and that the ubiquitinated disassembly products are carried to the cell body through IFT (Huang et al., 2009). Moreover, the USP plays a critical role in signaling pathways controlled by the primary cilium, including the Sonic hedgehog, Notch and Wnt pathways, which involve the proteolysis-mediated activation of downstream transcription factors (Gerhardt et al., 2016). Consistent with this function, several ciliary proteins, including Bardet Biedl syndrome (BBS) 4, oral-facial-digital syndrome 1 (OFD1) and RPGRIP1L, co-localize with and interact with components of the proteasome at the base of the cilium. Interestingly, the UPS-dependent paracrine signaling involving I $\mathrm{B}$ degradation and leading to activation of the transcription factor NF- $\mathrm{BB}$ was found to be controlled by BBS1, BBS4, and OFD1 (Liu et al., 2014), suggesting the possibility that ciliary proteins may have a role in cellular proteostasis beyond ciliary assembly/disassembly and signaling.

Interestingly, primary cilium assembly and function have been recently implicated in the other major proteostasis system of eukaryotic cells that is functionally interconnected with the UPS, namely autophagy. Here we will discuss the direct and indirect roles of the IFT system as a mediator of autophagy.

\section{Regulation of Autophagy by the IFT System}

Autophagy is a selective process exploited by all eukaryotic cells to regulate cytoplasmic turnover of proteins and organelles. While the final degradation step is mediated by the lysosome, autophagy can be distinguished in macroautophagy, microautophagy, and chaperone-mediated autophagy based on the mechanism of cargo targeting to this organelle (Parzych and Klionsky, 2014). During chaperone-mediated autophagy a protein translocation system localized at the lysosomal membrane regulates the transfer of cytosolic proteins to the lysosomes, while during microautophagy autophagic substrates are internalized through lysosomal membrane invaginations. Macroautophagy (commonly simply referred to as autophagy) is the best characterized form of autophagy. This is a vesicle-based process that involves the formation of the phagophore, which encloses the autophagic substrates to generate a double membrane vesicle, the autophagosome. Subsequently, the autophagosome fuses with the lysosome to form the autophagolysosome, where the lysosomal hydrolases allow for degradation of its contents. Finally, aminoacids and other degradation products are exported to the cytoplasm by 
lysosomal permeases and transporters and used by the cell for biosynthetic processes (Yu et al., 2018).

Autophagy has been shown to control ciliogenesis, as demonstrated pharmacologically or genetically by depletion of the autophagy protein ATG7 (Wang et al., 2015; Pampliega and Cuervo, 2016). IFT20 and the ciliopathy-related protein OFD1 are among the ciliogenesis proteins which have been identified as targets of autophagy (Pampliega et al., 2013; Tang et al., 2013). Under nutrient-rich conditions IFT20 is selectively degraded by basal autophagy, thereby preventing ciliary growth. Conversely, under conditions of serum deprivation IFT20 is spared from degradation while the ciliogenesis inhibitor OFD1 undergoes autophagic degradation, allowing for ciliary growth (Pampliega et al., 2013; Tang et al., 2013). Interestingly, the ciliary protein RPGRIP1L, which modulates the activity of the ciliary proteasome at the base of the cilium (Gerhardt et al., 2015), also controls autophagy by regulating the activity of mTOR (Struchtrup et al., 2018), thereby acting as a rheostat in the activation of the mutually exclusive ciliogenesis and autophagy pathways.

While autophagy is orchestrated by the autophagy-related (ATG) proteins (Hurley and Young, 2017), several ciliogenesis proteins, including the IFT proteins, have been demonstrated to participate in this process. Namely, IFT20 and IFT8 8 control serum starvation-induced autophagy in a pathway involving Hedgehog signaling by regulating the transport of several components of the autophagic machinery to the ciliary base (Pampliega et al., 2013). Indeed, the intracellular distribution of autophagy regulators, the PI3 kinase VPS34, the ATG proteins ATG16L, ATG5, ATG7 and ATG14, and the ubiquitin-like proteins MAP1LC3/LC3 (microtubule-associated protein 1 light chain 3) and GABARAP, was found to be altered in IFT $88^{-/-}$ mouse kidney epithelial cells, resulting in impaired autophagy (Pampliega et al., 2013). These results identify IFT proteins as regulators shared by the pathways that control autophagy and ciliogenesis and highlight a reciprocal interplay between these processes. Recruitment of the phagophore elongation complex component ATG16L to the basal body has also been observed in kidney tubular epithelial cells during fluid flow-induced autophagy (Orhon et al., 2016). The functional analysis of IFT2 $0^{-/-}$cells has led to the identification of IFT20 as responsible for this process. Indeed, based on coimmunoprecipitation and immunoimaging studies IFT20 was shown to interact with ATG16L and regulate its trafficking in association with carrier vesicles to the ciliary base (Pampliega et al., 2013), further underscoring the vesicular trafficking-related function of this IFT protein.

\section{IFT20 Controls Lysosome Biogenesis}

Autophagy is exploited by $\mathrm{T}$ cells not only to regulate $\mathrm{T}$ cell homeostasis but also to support metabolic functions essential for $\mathrm{T}$ cell development, activation and differentiation to effector and memory cells (Puleston and Simon, 2014; Dowling and Macian, 2018; Jacquin and Apetoh, 2018). We thus hypothesized that, if the autophagic function described for the IFT system in ciliated cells is conserved in $\mathrm{T}$ cells, we could expect autophagy defects to contribute to the $\mathrm{T}$ cell activation and differentiation abnormalities displayed by IFT20-deficient $\mathrm{T}$ cells (Finetti et al., 2009; Vivar et al., 2016). We therefore measured the autophagic degradation activity in T cells depleted of IFT20, both under conditions of starvation and under nutrient and growth factor-rich conditions, using the standard immunoblot-based autophagy assay to measure the generation of LC3-II, the cleaved and lipidated form of LC3 that is associated with autophagosomal membranes (Klionsky et al., 2016). The results showed an autophagy defect, indicating that the autophagy-related function of IFT20 extends to the non-ciliated T cells (Finetti et al., 2019). Interestingly, an accumulation of LC3II could be detected in IFT20-deficient $\mathrm{T}$ cells under basal conditions (Finetti et al., 2019). Since LC3-II is degraded when autophagosomes fuse with lysosomes, this suggested the possibility of a defect in the late steps of the autophagy pathway in these cells. Indeed, imaging studies revealed that LC3-II accumulated in autolysosomes in IFT20-depleted $\mathrm{T}$ cells, concomitant with the lysosomal accumulation of lipid droplets, which similar to LC3-II are degraded by lysosomal hydrolases (Singh et al., 2009; Platt et al., 2012; Yu et al., 2018). These results pointed to a lysosomal defect, one consequence of which would be impaired autophagy.

IFT20 deficiency in T cells resulted in a peculiar lysosome phenotype, with a decrease in their number which was paralleled by an increase in their size. This is the typical phenotype observed with dysfunctional lysosomes that become engorged by undigested material. Under these conditions the cell attempts to compensate by promoting the biogenesis of new lysosomes through the activation of the CLEAR (coordinated lysosomal expression and regulation) gene network. This includes all the genes that encode the structural and effector components of the lysosomes and is controlled by the master transcription factor TFEB (Garbi et al., 2010; Palmieri et al., 2011; Settembre et al., 2011). In support of a lysosomal dysfunction, the expression of genes belonging to the CLEAR network was upregulated in IFT20-deficient $\mathrm{T}$ cells, including the integral lysosomal membrane proteins LAMP-1 and -2, the proteases cathepsin $\mathrm{B}$ and $\mathrm{D}$, the glycosaminoglycan-degrading enzyme hexosaminidase B, the lysosomal acid lipase and TFEB itself (Finetti et al., 2019).

Lysosome function depends on two main factors: acidification, which is mediated by the vacuolar ATPase and is essential for the activation of the lysosomal hydrolases, and transport of these hydrolases to the lysosomal lumen. Acidification was normal in IFT20-deficient $\mathrm{T}$ cells, as assessed using a $\mathrm{pH}$ sensitive probe. However, lysosomal protease activity was impaired, as measured using acid protease fluorescent probes, suggesting that the transport of the lysosomal hydrolases might be affected by IFT2 0 deficiency. In support of this notion, we found that lysosomes purified from IFT20-deficient $\mathrm{T}$ cells had a significant reduction in their acid hydrolase content (Finetti et al., 2019).

The intracellular trafficking of lysosomal enzymes relies on the activity of the cation-independent mannose 6-phosphate receptor (CI-MPR), a type-I 300-kDa transmembrane protein, which diverts these enzymes from the default secretory pathway by binding them, once they have been $\mathrm{N}$-glycosylated and then added with mannose-6-phosphate at the trans-Golgi network (TGN), where they are sorted into clathrin-coated vesicles. 


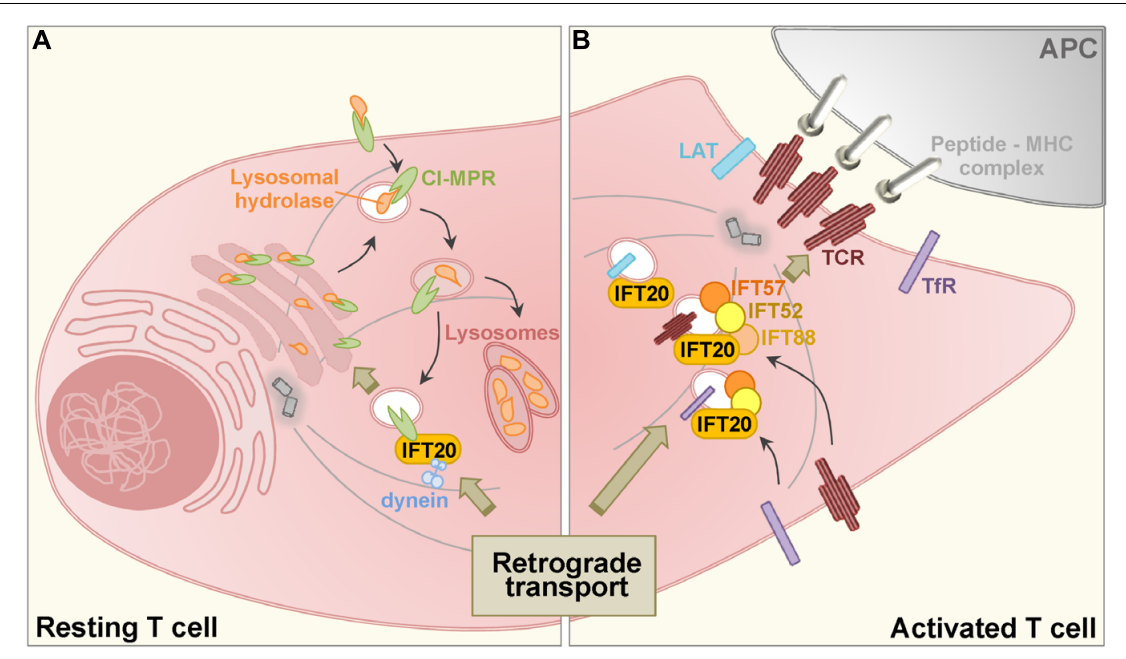

FIGURE 1 | Schematic representation of the role of IFT20 in the retrograde transport in resting (A) and activated (B) T cells. (A) Acid hydrolases are added with mannose 6-phosphate moieties at the trans-Golgi network and bind to the cation independent mannose-6-phosphate receptor (Cl-MPR) which is involved in their traffic from early to late endosomes (the decrease in $\mathrm{pH}$ is depicted by a color gradient, with darker vesicular lumen corresponding to the lowest $\mathrm{pH}$ ). Here, the acidic $\mathrm{pH}$ leads to the dissociation of the complex: hydrolases are delivered to the lysosomes, while Cl-MPRs recycle to the trans-Golgi through a retrograde transport pathway that requires IFT20 for coupling recycling Cl-MPRs to dynein. (B) When a T cell encounter with an antigen presenting cell (APC) expressing specific peptide-MHC complexes, engaged T cell antigen receptors (TCRs) induce the assembly of the immune synapse. TCR as well as the transferrin receptor (TfR) and the membrane-associated adaptor LAT polarize to the T cell: APC interface from an intracellular endosome-associated pool through a retrograde transport pathway that requires IFT proteins.

Vesicles transit through early endosomes to late endosomes, where the low $\mathrm{pH}$ allows for the dissociation of CI-MPRs from the hydrolases, which are released into lysosomes by fluid-phase transport, while CI-MPRs undergo retrograde traffic to the TGN for new cycles of transport (Braulke and Bonifacino, 2009; Lu and Hong, 2014). Using a specific antibody to tag CI-MPRs undergoing retrograde transport, we observed that these fail to efficiently recycle to the TGN in IFT20-deficient $\mathrm{T}$ cells and remain associated with endosomes dispersed away from the centrosome, implicating IFT20 in the retrograde transport of the CI-MPR (Finetti et al., 2019).

The CI-MPR retrieval pathway requires the retromer complex, which sequestrates the receptor into tubular endosomes, several Rab GTPases and their effectors, and the STX16/Vti1A/VAMP-3 SNARE complex, as well as the interaction with the dynein/dynactin minus-end directed microtubule motor complex (Hong et al., 2009; Wassmer et al., 2009; Cai et al., 2010; Pfeffer, 2011; Lu and Hong, 2014). Since one of the basic functions of the IFT system in ciliated cells is to couple cargo to molecular motors for transport along the axonemal microtubules we tested the association of IFT20 with dynein. We found that IFT20 co-precipitated and co-localized with dynein and the CI-MPR and that the ability of the CI-MPR to interact with dynein was impaired in IFT20-deficient T cells (Finetti et al., 2019).

Together, these findings indicate that IFT20 regulates CIMPR trafficking by coupling this receptor to dynein to allow for its retrograde transport to the TGN (Figure 1A). In its absence CI-MPRs accumulate in a dispersed traffic-incompetent endosomal compartment, thereby limiting the availability of CIMPRs at the TGN. This leads to a defect in the transport of acid hydrolases to lysosomes and impaired lysosome function. Additionally, these data highlight IFT20 as an adaptor implicated not only in endosome recycling to the plasma membrane, but also as a new component of the machinery responsible for retrograde transport to the TGN (Finetti et al., 2019). Consistent with this function, IFT20 participates in the endosome-mediated transport of the molecular adaptor LAT to the immune synapse (Vivar et al., 2016), which has recently been shown to depend on its Rab6-depedent retrograde transport to the Golgi apparatus (Carpier et al., 2018).

Of note, we have extended this study to B cells, which similar to $\mathrm{T}$ cells have no primary cilium, as well as to fibroblasts, which have a cilium. The lysosome-related defects observed on $\mathrm{T}$ cells were found to be shared by these cells when depleted of IFT20 (Finetti et al., 2019), underscoring the implication of IFT20 in a fundamental extraciliary function. Interestingly, in sperms from a male germ cell-specific IFT $20^{-/-}$mouse redundant cytoplasm was observed attached to the flagella, concomitant with a reduction in mature lysosomes, implicating IFT20 in the degradation of cytoplasmic components of the residual bodies in mouse testis (Zhang et al., 2016). Together, these data highlight a new cellular function of IFT20 in intracellular membrane trafficking related to the cellular degradation pathways that is conserved in ciliated and non-ciliated cells.

\section{CONCLUSION AND FUTURE DIRECTIONS}

The emerging scenario is that, by orchestrating intracellular vesicular trafficking, IFT proteins control crucial extraciliary 
processes spanning from endosome recycling to lysosome biogenesis, and function. These findings have important implications for the development of an effective immune response. We have previously reported that $\mathrm{T}$ cell activation and differentiation are impaired in T cells lacking IFT20 both in vitro and in vivo in a $\mathrm{T}$ cell-specific conditional knockout mouse and showed that this results from its ability to promote the polarized recycling to the immune synapse of endosome-associated TCR and LAT (Finetti et al., 2009; Vivar et al., 2016). The recent identification of IFT2 0 as a central regulator of lysosome function (Finetti et al., 2019) opens new future perspectives on its ability to control the immune response at a more global level. One central question is whether the lysosomal defect in IFT20-deficient T cells might impinge not only on the activation but also on the activity of cytotoxic $\mathrm{T}$ cells, which are responsible for the elimination of infected or cancer cells. Indeed, lytic granules are specialized lysosomes that exploit the CI-MPR pathway for the transport of the granzymes and possibly of perforin (Clark and Griffiths, 2003; Lopez et al., 2012), which mediate killing of their specific cell targets. Future studies will be required to evaluate the implication of IFT2 0 in the biogenesis of these organelles in cytotoxic T cells as well as in NK cells, their innate cytotoxic counterparts. Another level at which IFT20 may indirectly impact on the generation of $\mathrm{T}$ cell specific immunity through its lysosome-related function is by modulating the ability of antigen presenting cells to activate $\mathrm{T}$ cells. Indeed, antigen processing and presentation through the MHCII pathway, on which the generation of $\mathrm{T}$ cell-mediated immunity is critically dependent, also requires functional lysosomes, suggesting that IFT20 might participate in this process. Finally, lysosomal integrity has been demonstrated to be required for efficient extraction of surfacetethered antigens at the synapse formed by B cells with follicular macrophages, as this process relies on the local secretion of lysosomes that release lytic enzymes and acidify the synaptic cleft, allowing for antigen extraction (Yuseff et al., 2013; Sáez et al., 2019). Hence, by modulating lysosome function IFT20 may also participate in B cell activation. These considerations suggest new exciting directions to be tested experimentally to

\section{REFERENCES}

Bizet, A. A., Becker-Heck, A., Ryan, R., Weber, K., Filhol, E., Krug, P., et al. (2015). Mutations in TRAF3IP1/IFT54 reveal a new role for IFT proteins in microtubule stabilization. Nat. Commun. 6:8666. doi: 10.1038/ncomms9666

Borovina, A., and Ciruna, B. (2013). IFT88 plays a Cilia- and PCP-independent role in controlling oriented cell divisions during vertebrate embryonic development. Cell Rep. 5, 37-43. doi: 10.1016/j.celrep.2013.08.043

Boukhalfa, A., Miceli, C., Ávalos, Y., Morel, E., and Dupont, N. (2019). Interplay between primary cilia, ubiquitin-proteasome system and autophagy. Biochimie 166, 286-292. doi: 10.1016/j.biochi.2019.06.009

Braulke, T., and Bonifacino, J. S. (2009). Sorting of lysosomal proteins. Biochim. Biophys. Acta Mol. Cell Res. 1793, 605-614. doi: 10.1016/J.BBAMCR.2008.10. 016

Cai, Q., Lu, L., Tian, J.-H., Zhu, Y.-B., Qiao, H., and Sheng, Z.-H. (2010). Snapinregulated late endosomal transport is critical for efficient autophagy-lysosomal function in neurons. Neuron 68, 73-86. doi: 10.1016/j.neuron.2010.09.022

Carpier, J.-M., Zucchetti, A. E., Bataille, L., Dogniaux, S., Shafaq-Zadah, M., Bardin, S., et al. (2018). Rab6-dependent retrograde traffic of LAT controls elucidate the role of IFT20 and potentially other IFT proteins that physically and/or functionally interact with IFT20 in the lysosomal control of the immune response. Additionally, these observations highlight the potential involvement of IFT proteins in $\mathrm{T}$ and $\mathrm{B}$ cell-related immunodeficiency disorders of unknown etiology and also underscore the importance to search for the presence of immune dysfunctions in ciliopathies that have been linked to mutations in components of the IFT system or of other ciliary proteins implicated in the IFT-dependent pathways of lymphocyte activation.

Another important implication of the lysosome-related function of IFT20 and, at a more general level, of the extraciliary functions identified to date for ciliary proteins, is that the complex multiple abnormalities observed in the wide range of known ciliopathies and attributed to defects in cilia assembly and/or function may need to be reconsidered at the light of these functions. In this respect, the non-ciliated immune cells represent a perfect system to study cilium-independent cellular functions.

\section{AUTHOR CONTRIBUTIONS}

All authors listed have made a substantial, direct and intellectual contribution to the work, and approved it for publication. FF, NC, and $\mathrm{CB}$ wrote the manuscript. FF prepared the figure.

\section{FUNDING}

Part of the work discussed in this review was carried out with the support of Fondazione Telethon, Italy (Grant GGP16003) and AIRC (Grant IG 20148) to CB.

\section{ACKNOWLEDGMENTS}

The authors wish to thank Mike Dustin and Claire Hivroz for their stimulating discussions.

immune synapse formation and T cell activation. J. Exp. Med. 215, 1245-1265. doi: $10.1084 /$ jem.20162042

Cassioli, C., and Baldari, C. T. (2019). A ciliary view of the immunological synapse. Cells 8:789. doi: 10.3390/cells8080789

Clark, R., and Griffiths, G. M. (2003). Lytic granules, secretory lysosomes and disease. Curr. Opin. Immunol. 15, 516-521. doi: 10.1016/S0952-7915(03)001134

Delaval, B., Bright, A., Lawson, N. D., and Doxsey, S. (2011). The cilia protein IFT88 is required for spindle orientation in mitosis. Nat. Cell Biol. 13, 461-468. doi: $10.1038 /$ ncb2202

Dowling, S. D., and Macian, F. (2018). Autophagy and T cell metabolism. Cancer Lett. 419, 20-26. doi: 10.1016/j.canlet.2018.01.033

Dustin, M. L., and Choudhuri, K. (2016). Signaling and polarized communication across the T Cell immunological synapse. Annu. Rev. Cell Dev. Biol. 32, 303-325. doi: 10.1146/annurev-cellbio-100814- 125330

Finetti, F., Cassioli, C., Cianfanelli, V., Onnis, A., Paccagnini, E., Kabanova, A., et al. (2019). The intraflagellar transport protein IFT20 controls lysosome biogenesis by regulating the post-Golgi transport of acid hydrolases. Cell Death Differ. 29:13. doi: 10.1038/s41418-019-0357-y 
Finetti, F., Paccani, S. R., Riparbelli, M. G., Giacomello, E., Perinetti, G., Pazour, G. J., et al. (2009). Intraflagellar transport is required for polarized recycling of the TCR/CD3 complex to the immune synapse. Nat. Cell Biol. 11, 1332-1339. doi: $10.1038 /$ ncb 1977

Finetti, F., Patrussi, L., Masi, G., Onnis, A., Galgano, D., Lucherini, O. M., et al. (2014). Specific recycling receptors are targeted to the immune synapse by the intraflagellar transport system. J. Cell Sci. 127, 1924-1937. doi: 10.1242/jcs. 139337

Follit, J. A., San Agustin, J. T., Xu, F., Jonassen, J. A., Samtani, R., Lo, C. W., et al. (2008). The Golgin GMAP210/TRIP11 anchors IFT20 to the Golgi complex. PLoS Genet. 4:e1000315. doi: 10.1371/journal.pgen.1000315

Follit, J. A., Tuft, R. A., Fogarty, K. E., and Pazour, G. J. (2006). The intraflagellar transport protein IFT20 is associated with the golgi complex and is required for cilia assembly. Mol. Biol. Cell 17, 3781-3792. doi: 10.1091/mbc.e06-02-0133

Garbi, N., Hämmerling, G. J., Probst, H.-C., and van den Broek, M. (2010). Tonic $\mathrm{T}$ cell signalling and $\mathrm{T}$ cell tolerance as opposite effects of self-recognition on dendritic cells. Curr. Opin. Immunol. 22, 601-608. doi: 10.1016/j.coi.2010.08. 007

Gerhardt, C., Leu, T., Lier, J. M., and Rüther, U. (2016). The cilia-regulated proteasome and its role in the development of ciliopathies and cancer. Cilia 5:14. doi: 10.1186/s13630-016-0035-3

Gerhardt, C., Lier, J. M., Burmühl, S., Struchtrup, A., Deutschmann, K., Vetter, M., et al. (2015). The transition zone protein Rpgripll regulates proteasomal activity at the primary cilium. J. Cell Biol. 210, 115-133. doi: 10.1083/jcb.201408060

Gonçalves, J., and Pelletier, L. (2017). The ciliary transition zone: finding the pieces and assembling the gate. Mol. Cells 40, 243-253. doi: 10.14348/molcells.2017. 0054

Hong, Z., Yang, Y., Zhang, C., Niu, Y., Li, K., Zhao, X., et al. (2009). The retromer component SNX6 interacts with dynactin p150Glued and mediates endosome-to-TGN transport. Cell Res. 19, 1334-1349. doi: 10.1038/cr.2009.130

Hsiao, Y.-C., Tuz, K., and Ferland, R. J. (2012). Trafficking in and to the primary cilium. Cilia 1:4. doi: 10.1186/2046-2530-1-4

Hua, K., and Ferland, R. J. (2018). Primary cilia proteins: ciliary and extraciliary sites and functions. Cell. Mol. Life Sci. 75, 1521-1540. doi: 10.1007/s00018-0172740-5

Huang, K., Diener, D. R., and Rosenbaum, J. L. (2009). The ubiquitin conjugation system is involved in the disassembly of cilia and flagella. J. Cell Biol. 186, 601-613. doi: 10.1083/jcb.200903066

Hurley, J. H., and Young, L. N. (2017). Mechanisms of autophagy initiation. Annu. Rev. Biochem. 86, 225-244. doi: 10.1146/annurev-biochem-061516-044820

Jacquin, E., and Apetoh, L. (2018). Cell-Intrinsic roles for autophagy in modulating CD4 T cell functions. Front. Immunol. 9:1023. doi: 10.3389/fimmu.2018.01023

Jékely, G., and Arendt, D. (2006). Evolution of intraflagellar transport from coated vesicles and autogenous origin of the eukaryotic cilium. Bioessays 28, 191-198. doi: 10.1002/bies.20369

Jonassen, J. A., Agustin, J. S., Follit, J. A., and Pazour, G. J. (2008). Deletion of IFT20 in the mouse kidney causes misorientation of the mitotic spindle and cystic kidney disease. J. Cell Biol. 183, 377-384. doi: 10.1083/jcb.200808137

Kasahara, K., Kawakami, Y., Kiyono, T., Yonemura, S., Kawamura, Y., Era, S., et al. (2014). Ubiquitin-proteasome system controls ciliogenesis at the initial step of axoneme extension. Nat. Commun. 5:5081. doi: 10.1038/ncomms6081

Klionsky, D. J., Abdelmohsen, K., Abe, A., Abedin, M. J., Abeliovich, H., Arozena, A. A., et al. (2016). Guidelines for the use and interpretation of assays for monitoring autophagy (3rd edition). Autophagy 12, 1-222. doi: 10.1080/ 15548627.2015.1100356

Kozminski, K. G., Beech, P. L., and Rosenbaum, J. L. (1995). The Chlamydomonas kinesin-like protein FLA10 is involved in motility associated with the flagellar membrane. J. Cell Biol. 131, 1517-1527. doi: 10.1083/jcb.131.6.1517

Liu, Y. P., Tsai, I.-C., Morleo, M., Oh, E. C., Leitch, C. C., Massa, F., et al. (2014). Ciliopathy proteins regulate paracrine signaling by modulating proteasomal degradation of mediators. J. Clin. Invest. 124, 2059-2070. doi: 10.1172/ JCI71898

Lopez, J. A., Brennan, A. J., Whisstock, J. C., Voskoboinik, I., and Trapani, J. A. (2012). Protecting a serial killer: pathways for perforin trafficking and selfdefence ensure sequential target cell death. Trends Immunol. 33, 406-412. doi: 10.1016/j.it.2012.04.001

Lu, L., and Hong, W. (2014). From endosomes to the trans-Golgi network. Semin. Cell Dev. Biol. 31, 30-39. doi: 10.1016/j.semcdb.2014.04.024
Nishita, M., Park, S. Y., Nishio, T., Kamizaki, K., Wang, Z., Tamada, K., et al. (2017). Ror2 Signaling regulates Golgi structure and transport through IFT20 for tumor invasiveness. Sci. Rep. 7:1. doi: 10.1038/s41598-016-0028-x

Onnis, A., Finetti, F., and Baldari, C. T. (2016). Vesicular trafficking to the immune synapse: how to assemble receptor-tailored pathways from a basic building set. Front. Immunol. 7:50. doi: 10.3389/fimmu.2016.00050

Orhon, I., Dupont, N., Zaidan, M., Boitez, V., Burtin, M., Schmitt, A., et al. (2016). Primary-cilium-dependent autophagy controls epithelial cell volume in response to fluid flow. Nat. Cell Biol. 18, 657-667. doi: 10.1038/ncb3360

Palmieri, M., Impey, S., Kang, H., di Ronza, A., Pelz, C., Sardiello, M., et al. (2011). Characterization of the CLEAR network reveals an integrated control of cellular clearance pathways. Hum. Mol. Genet. 20, 3852-3866. doi: 10.1093/ hmg/ddr306

Pampliega, O., and Cuervo, A. M. (2016). Autophagy and primary cilia: dual interplay. Curr. Opin. Cell Biol. 39, 1-7. doi: 10.1016/j.ceb.2016.01.008

Pampliega, O., Orhon, I., Patel, B., Sridhar, S., Díaz-Carretero, A., Beau, I., et al. (2013). Functional interaction between autophagy and ciliogenesis. Nature 502, 194-200. doi: 10.1038/nature12639

Parzych, K. R., and Klionsky, D. J. (2014). An overview of autophagy: morphology, mechanism, and regulation. Antioxid. Redox Signal. 20, 460-473. doi: 10.1089/ ars.2013.5371

Pedersen, L. B., Mogensen, J. B., and Christensen, S. T. (2016). Endocytic control of cellular signaling at the primary cilium. Trends Biochem. Sci. 41, 784-797. doi: 10.1016/j.tibs.2016.06.002

Pedersen, L. B., and Rosenbaum, J. L. (2008). Chapter two intraflagellar transport (IFT). Role in Ciliary Assembly, Resorption and Signalling. Curr. Top. Dev. Biol. 85, 23-61. doi: 10.1016/S0070-2153(08)00802-8

Pfeffer, S. R. (2011). Entry at the trans-face of the Golgi. Cold Spring Harb. Perspect. Biol. 3:a005272. doi: 10.1101/cshperspect.a005272

Platt, F. M., Boland, B., and van der Spoel, A. C. (2012). The cell biology of disease: lysosomal storage disorders: the cellular impact of lysosomal dysfunction. J. Cell Biol. 199, 723. doi: 10.1083/JCB.201208152

Prevo, B., Scholey, J. M., and Peterman, E. J. G. (2017). Intraflagellar transport: mechanisms of motor action, cooperation, and cargo delivery. FEBS J. 284, 2905-2931. doi: 10.1111/febs.14068

Puleston, D. J., and Simon, A. K. (2014). Autophagy in the immune system. Immunology 141, 1-8. doi: 10.1111/imm.12165

Qin, H., Wang, Z., Diener, D., and Rosenbaum, J. (2007). Intraflagellar transport protein 27 is a small g protein involved in cell-cycle control. Curr. Biol. 17, 193-202. doi: 10.1016/J.CUB.2006.12.040

Robert, A., Margall-Ducos, G., Guidotti, J.-E., Brégerie, O., Celati, C., Bréchot, C., et al. (2007). The intraflagellar transport component IFT88/polaris is a centrosomal protein regulating G1-S transition in non-ciliated cells. J. Cell Sci. 120, 918. doi: 10.1242/jcs.03422

Sáez, J. J., Diaz, J., Ibañez, J., Bozo, J. P., Cabrera Reyes, F., Alamo, M., et al. (2019). The exocyst controls lysosome secretion and antigen extraction at the immune synapse of B cells. J. Cell Biol. 218, 2247-2264. doi: 10.1083/jcb.201811131

Satir, P., Mitchell, D. R., and Jékely, G. (2008). How did the cilium evolve? Curr. Top. Dev. Biol. 85, 63-82. doi: 10.1016/S0070-2153(08)00803-X

Sedmak, T., and Wolfrum, U. (2010). Intraflagellar transport molecules in ciliary and nonciliary cells of the retina. J. Cell Biol. 189, 171-186. doi: 10.1083/jcb. 200911095

Settembre, C., Di Malta, C., Polito, V. A., Garcia Arencibia, M., Vetrini, F., Erdin, S., et al. (2011). TFEB links autophagy to lysosomal biogenesis. Science 332, 1429-1433. doi: 10.1126/science. 1204592

Singh, R., Kaushik, S., Wang, Y., Xiang, Y., Novak, I., Komatsu, M., et al. (2009). Autophagy regulates lipid metabolism. Nature 458, 1131-1135. doi: 10.1038/ nature 07976

Spektor, A., Tsang, W. Y., Khoo, D., and Dynlacht, B. D. (2007). Cep97 and CP110 suppress a cilia assembly program. Cell 130, 678-690. doi: 10.1016/j.cell.2007. 06.027

Struchtrup, A., Wiegering, A., Stork, B., Rüther, U., and Gerhardt, C. (2018). The ciliary protein RPGRIP1L governs autophagy independently of its proteasomeregulating function at the ciliary base in mouse embryonic fibroblasts. Autophagy 14:567. doi: 10.1080/15548627.2018.1429874

Tang, Z., Lin, M. G., Stowe, T. R., Chen, S., Zhu, M., Stearns, T., et al. (2013). Autophagy promotes primary ciliogenesis by removing OFD1 from centriolar satellites. Nature 502, 254-257. doi: 10.1038/nature12606 
Taschner, M., and Lorentzen, E. (2016). The intraflagellar transport machinery. Cold Spring Harb. Perspect. Biol. 8:a028092. doi: 10.1101/cshperspect.a028092

Taulet, N., Vitre, B., Anguille, C., Douanier, A., Rocancourt, M., Taschner, M., et al. (2017). IFT proteins spatially control the geometry of cleavage furrow ingression and lumen positioning. Nat. Commun. 8:1928. doi: 10.1038/s41467017-01479-3

Tuz, K., Bachmann-Gagescu, R., O’Day, D. R., Hua, K., Isabella, C. R., Phelps, I. G., et al. (2014). Mutations in CSPP1 cause primary cilia abnormalities and joubert syndrome with or without jeune asphyxiating thoracic dystrophy. Am. J. Hum. Genet. 94, 62-72. doi: 10.1016/j.ajhg.2013.11.019

Vivar, O. I., Masi, G., Carpier, J., Magalhaes, J. G., Galgano, D., Pazour, G. J., et al. (2016). IFT20 controls LAT recruitment to the immune synapse and T-cell activation in vivo. Proc. Natl. Acad. Sci. U.S.A. 113, 386-391. doi: 10.1073/pnas. 1513601113

Wang, S., Livingston, M. J., Su, Y., and Dong, Z. (2015). Reciprocal regulation of cilia and autophagy via the MTOR and proteasome pathways. Autophagy 11, 607-616. doi: 10.1080/15548627.2015.1023983

Wang, Z., Wann, A. K. T., Thompson, C. L., Hassen, A., Wang, W., and Knight, M. M. (2016). IFT88 influences chondrocyte actin organization and biomechanics. Osteoarthr. Cartil. 24, 544-554. doi: 10.1016/j.joca.2015.10.003

Wassmer, T., Attar, N., Harterink, M., van Weering, J. R. T., Traer, C. J., Oakley, J., et al. (2009). The retromer coat complex coordinates endosomal sorting and dynein-mediated transport, with carrier recognition by the trans-Golgi network. Dev. Cell 17, 110-122. doi: 10.1016/j.devcel.2009. 04.016
Wood, C. R., Wang, Z., Diener, D., Zones, J. M., Rosenbaum, J., and Umen, J. G. (2012). IFT proteins accumulate during cell division and localize to the cleavage furrow in chlamydomonas. PLoS One 7:e30729. doi: 10.1371/journal. pone.0030729

Yu, L., Chen, Y., and Tooze, S. A. (2018). Autophagy pathway: cellular and molecular mechanisms. Autophagy 14, 207-215. doi: 10.1080/15548627.2017. 1378838

Yuseff, M.-I., Pierobon, P., Reversat, A., and Lennon-Duménil, A.-M. (2013). How B cells capture, process and present antigens: a crucial role for cell polarity. Nat. Rev. Immunol. 13, 475-486. doi: 10.1038/nri3469

Zhang, Z., Li, W., Zhang, Y., Zhang, L., Teves, M. E., and Liu, H. (2016). Intraflagellar transport protein IFT20 is essential for male fertility and spermiogenesis in mice. Mol. Biol. Cell 27, 3705-3716. doi: 10.1091/mbc.e1605-0318

Conflict of Interest: The authors declare that the research was conducted in the absence of any commercial or financial relationships that could be construed as a potential conflict of interest.

Copyright (C) 2019 Finetti, Capitani and Baldari. This is an open-access article distributed under the terms of the Creative Commons Attribution License (CC BY). The use, distribution or reproduction in other forums is permitted, provided the original author(s) and the copyright owner(s) are credited and that the original publication in this journal is cited, in accordance with accepted academic practice. No use, distribution or reproduction is permitted which does not comply with these terms. 